\title{
Indirect Combustion Noise Generation in Gas Turbines
}

\author{
F. Bake* , U. Michel ${ }^{\dagger}$ and I. Röhle \\ German Aerospace Center, Institute of Propulsion Technology, Berlin, Germany \\ C. Richter ${ }^{\S}$ and F. Thiele \\ Berlin University of Technology, Hermann-Föttinger-Institute of Fluid Mechanics, Germany \\ M. Liull and B. Noll** \\ German Aerospace Center, Institute of Combustion Technology, Stuttgart, Germany
}

\begin{abstract}
The paper investigates the indirect combustion noise, which is generated during the acceleration of the convected entropy nonuniformities of the combustion products in the outlet nozzle of the combustion chamber. The generation mechanism of the indirect noise is proven experimentally and through numerical simulation. Probe microphones and fast thermocouple probes were used to measure pressure and temperature fluctuations. The generation of indirect noise is verified via the phase relationship between thermocouple and microphone signals. The flow field in the combustion chamber is simulated by means of an unsteady RANS computation. Self excited oscillations are used for the computation of the direct and indirect noise generation of the combustion chamber. Since the related frequencies are low and the corresponding scales much larger than the turbulent scales, a CAA-method is employed for both the propagation of sound waves as well as entropy perturbations. It is shown that the CAA method is capable to describe the acoustical properties of the combustion system found in the experiments when the URANS simulation is used as input. The experimental results also show that indirect combustion noise may contain high frequency noise contributions, which are generally attributed to turbine noise.
\end{abstract}

\section{Introduction}

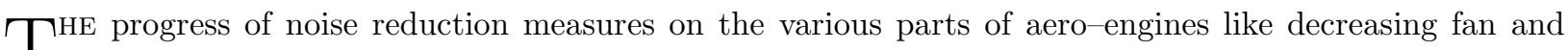
1 jet-mixing noise enhances the relative importance of combustion noise with respect to the total aircraft noise in particular during the landing approach. The total noise radiated by a combustion chamber system consists of direct and indirect combustion noise like shown in a generalized acoustic energy equation by Dowling ${ }^{1}$. The direct noise sources are related to the unsteady combustion process itself, e.g. to unsteady heat release. ${ }^{2}$ The indirect combustion noise is generated when fluid with a nonuniform entropy distribution is accelerated in or convected through the nozzle, which is located at the downstream end of the combustion chamber. In gas turbines, the inlet guide vanes for the first turbine stage serve as nozzle for the combustion chamber. The flow in this nozzle is choked in aero-engines in practically all relevant operating conditions. The underlying theory of these acoustic mechanisms is described by Marble \& Candel $^{3}$. The separation of hydrodynamic, entropy and combustion noise was investigated earlier via cross-spectral analysis. ${ }^{4}$ However, the contribution of indirect combustion noise to the total noise emission of aero-engine combustors is not known.

\footnotetext{
*Research Assistant, Müller-Breslau-Str. 8, 10623 Berlin, Germany, AIAA Student Member; Experiments, Email: Friedrich.Bake@dlr.de

†Senior Scientist, Müller-Breslau-Str. 8, 10623 Berlin, Germany, AIAA Member; Experiments.

${ }^{\ddagger}$ Research Scientist, Müller-Breslau-Str. 8, 10623 Berlin, Germany; Experiments.

$\S$ Research Assistant, Müller-Breslau-Str. 8, 10623 Berlin, Germany, AIAA Student Member; CAA.

ๆ Professor, Müller-Breslau-Str. 8, 10623 Berlin, Germany, AIAA Member; CAA.

\|Research Assistant, Pfaffenwaldring 38-40, 70569 Stuttgart, Germany; CFD.

** Reasearch Scientist, Pfaffenwaldring 38-40, 70569 Stuttgart, Germany; CFD.
} 
In order to explore the generation mechanism and to evaluate the relevance of indirect combustion noise a model combustor flow is investigated by experimental means and numerical simulation.

\section{Experimental Study}

The experimental investigations focus primarily on verifying the relevance of indirect combustion noise generation in aero-engine combustors. Furthermore, the experimental results are used to validate the noise predictions of the hybrid numerical simulations.

\section{A. Test Facility}

The setup for the experimental investigation is carefully chosen to replicate combustion flow characteristics of full scale gas turbines while still permitting analysis by experimental means. In order to simplify the numerical approach the system is designed axisymmetrically.
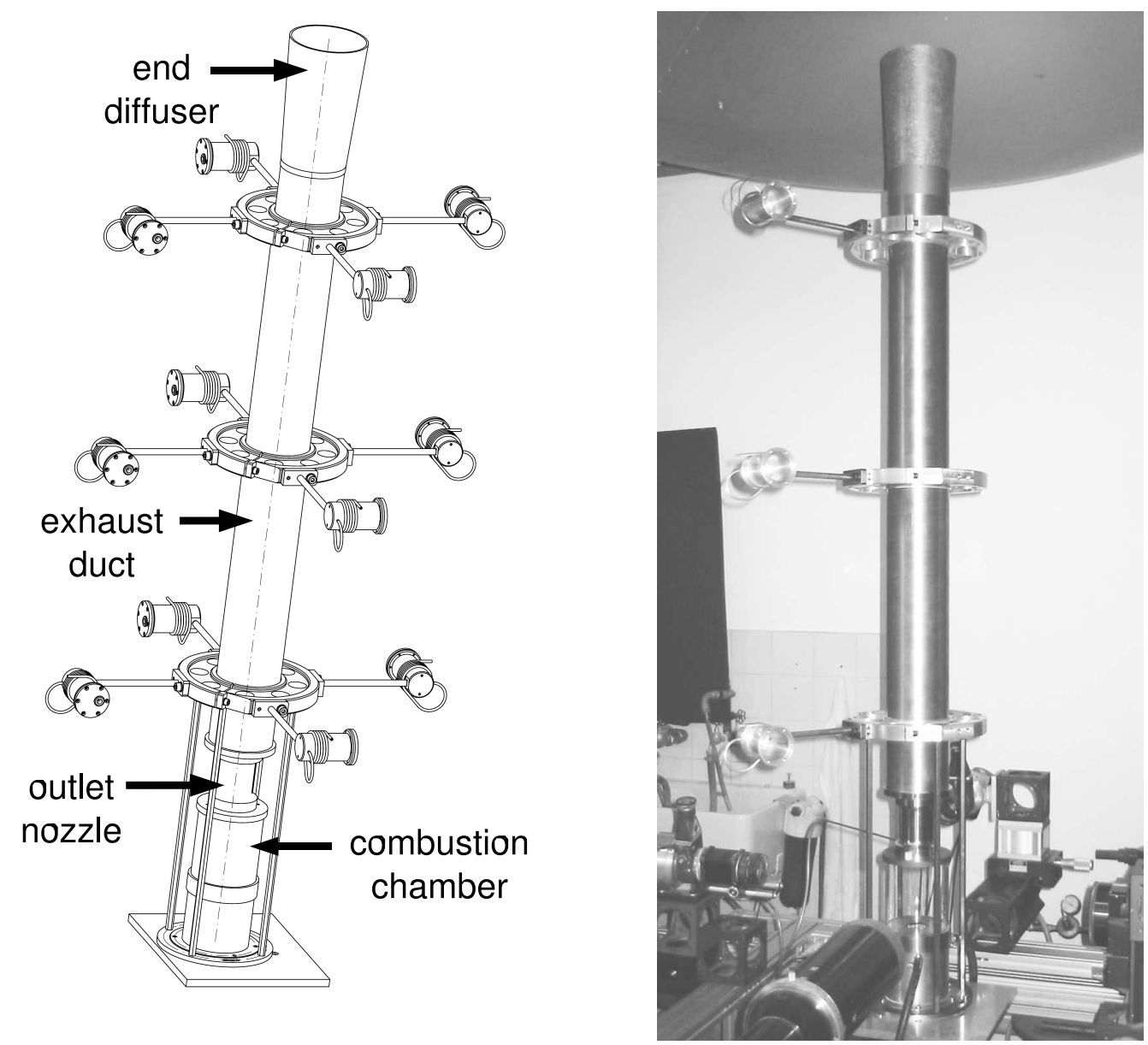

Figure 1. Isometric view and photo of the experimental facility.

The test rig, shown in figure 1 consists of three sections: combustion chamber, outlet nozzle and exhaust duct. A burner with a swirled dual air-flow nozzle is used to stabilize the combustion zone. 
A sketch of the burner in figure 2 illustrates the inner and the outer co-rotating air-flows, which are both fed from a common plenum chamber. The inner air nozzle has a diameter of $15 \mathrm{~mm}$ and the outer annular air nozzle has 17 and $25 \mathrm{~mm}$ inner and outer diameters, respectively. Methane gas is introduced as combustible through circular orfices that are arranged along an annular ring between the two air streams.

The combustion chamber itself is made of either a fused quartz glass or a stainless steel cylinder with $100 \mathrm{~mm}$ inner diameter. The chamber has a length of $113 \mathrm{~mm}$ and is terminated by a convergent-

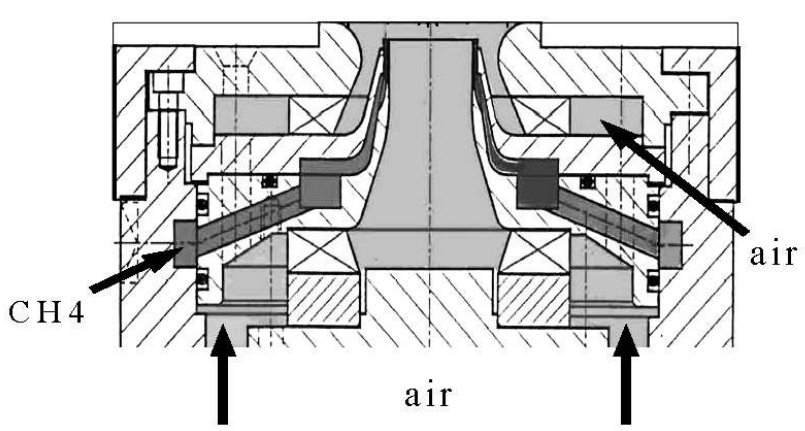

Figure 2. Layout of the dual swirl nozzle. divergent nozzle as shown in figure 3 . The outlet nozzle can be exchanged with nozzles of different throat diameters for testing the influence of the outlet Mach number on the indirect combustion noise.

The outlet nozzle is attached to an exhaust duct with the same diameter as the combustion chamber. In order to reduce the acoustic impedance jump at the exhaust outlet, a diffuser is attached at the end. (see figure 1). In addition, the diffuser is perforated with holes of $2 \mathrm{~mm}$ diameter with increasing perforation density towards the exit. The objective of this exhaust duct termination was to reduce acoustic reflections in order to reduce the influence of these reflections on the combustion process. The termination is designed in consideration of the results of Shenoda. ${ }^{5}$ The combustion noise emission is studied in the exhaust duct with the aid of probe microphones, which can be seen in figure 1 .

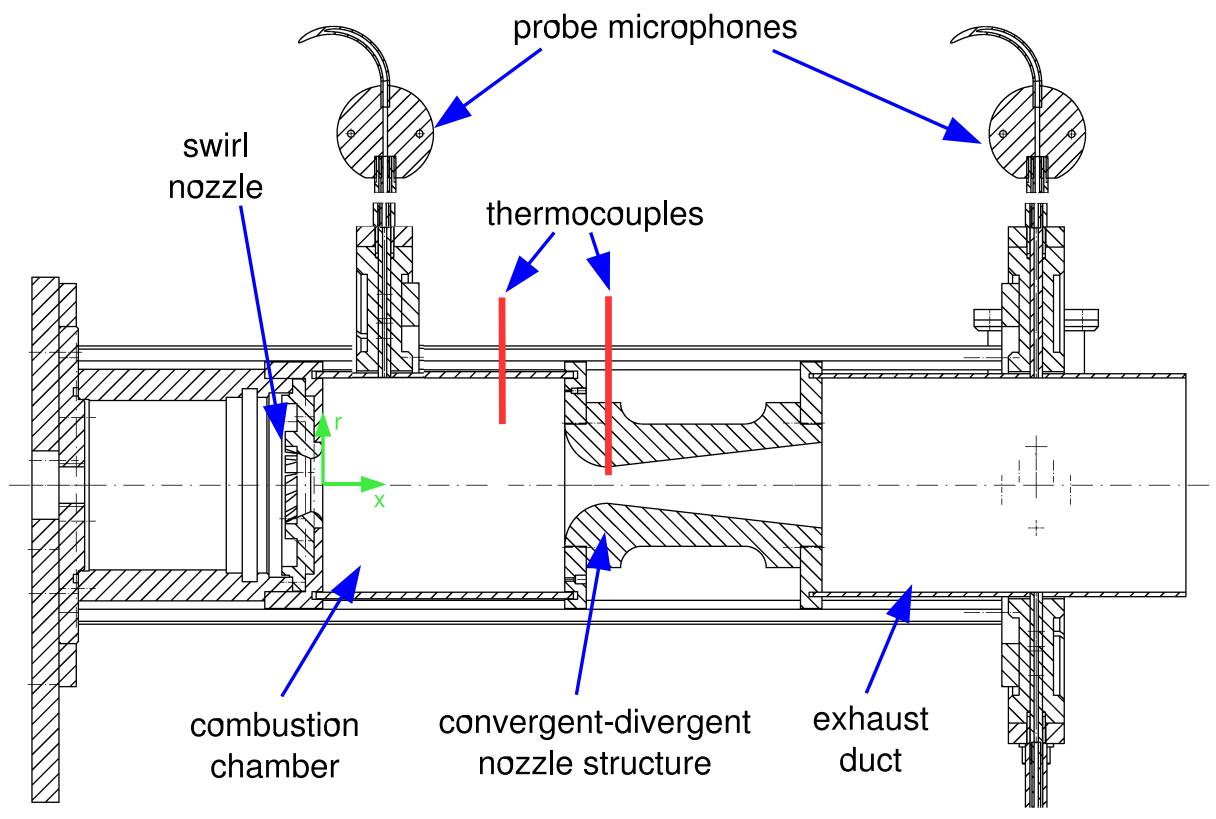

Figure 3. Sketch of the combustion chamber setup used for the model experiments.

The coordinate system of this flow configuration is indicated in figure 3 (green arrows) where the origin is set in the outlet plane of the swirl burner nozzle on the center axis of the combustion chamber.

In order to excite the combustion process with non-periodic perturbations, a piezoelectric device is installed in the fuel gas supply system to modulate the fuel mass flux dynamically. The fuel gas modulator is driven by a frequency generator. 


\section{B. Diagnostics}

Sound pressure measurements in combustion environments put high demands on the acoustic equipment. High temperatures up to $2000 \mathrm{~K}$ and highly corrosive exhaust gases disallow the usage of normal microphone setups. To prevent sensor destruction a probe microphone configuration shown in figure 4 is used. It can be operated up to the mean pressures experienced in operating gas turbines.

Due to the spatial separation between the measurement location at the walls of the combustion chamber or the exhaust duct and the microphone itself, common 1/4-inch-microphones can be used in experiments at ambient pressures. The connection between the exhaust duct wall and the microphone is realized by a steel tube of $2 \mathrm{~mm}$ inner diameter. For impedance matching and to avoid standing-wave effects in the probe tube, this is extended according to the principle of the semi-infinite acoustic duct.

The microphone (GRAS 40BH) is located inside the cylindrical pressure chamber shown in figure 4 and is connected to the probe tube via a pin hole. The probe tube is purged with Nitrogen from the rear end with a well controlled small flow rate, which is almost independent of the mean pressure inside the combustion chamber. The purging prevents the diaphragm of the microphone from damage by corrosive combustion products. The phase and amplitude of the collected data are corrected based on a comparison at ambient temperatures with flush mounted

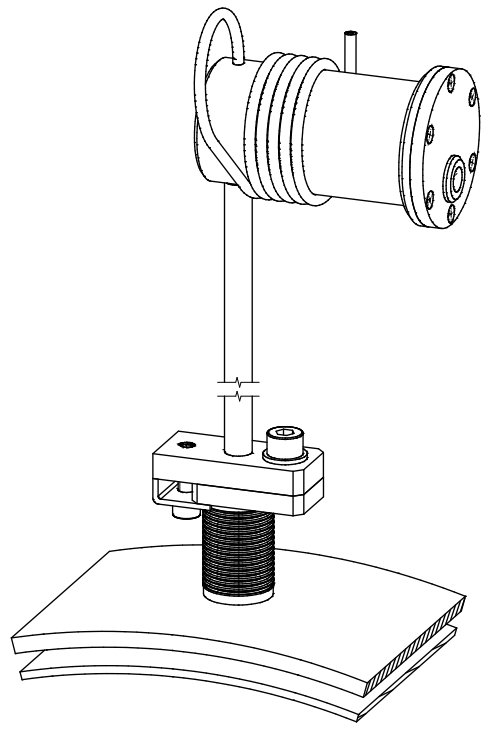

Figure 4. Sketch of the probe microphone microphones.

In the current setup the combustion chamber can be equipped with up to three probe microphones. On the exhaust duct system twelve microphones can be installed at three axial and four circumferential positions. From the calibrated time-series, the downstream and upstream propagating acoustical radial duct modes are recalculated using an inhouse processing code ${ }^{6,}{ }^{7}$ In the considered frequency range, the plane wave modes are the only propagating modes.

In order to gain information about convecting temperature fluctuations or so called entropy waves, bare wire thermocouples are installed in the combustion chamber and in the throat of the outlet nozzle.

For validation purpose and to map the flow field topology, the flow velocity field was measured using a quartz glass combustion chamber with square cross section and a three-component Laser-Doppler-System.

\section{Results}

The existence of entropy noise is demonstrated in three different ways:

1. by considering the phase relationships between the thermocouple signal from the combustion chamber and the acoustic signals from either the combustion chamber or the exhaust duct,

2. by analyzing phase averaged time series of these signals in a non-periodic excitation mode of the combustion system,

3. by comparing the power spectra of the probe-microphone signals from the combustion chamber and the exhaust pipe.

\section{Phase Relationship}

The propagation speed of traveling perturbations measured by certain sensors at different positions can be determined from the phases of the cross spectra between the signals of these sensors.

The combustion chamber setup was operated in a resonant self-oscillating mode and signals from a thermocouple in the combustion chamber as well as microphone signals from the combustion chamber and 


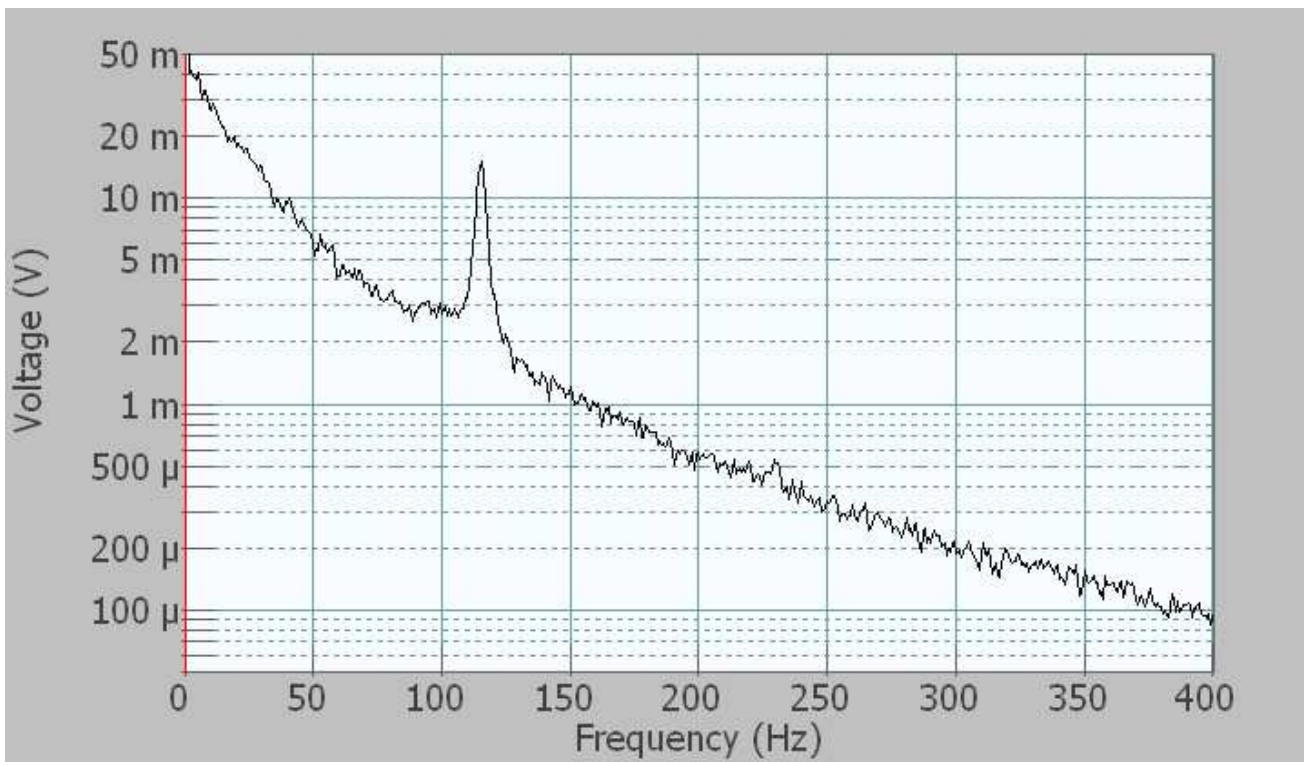

Figure 5. Power spectrum of the thermocouple signal in the combustion chamber in a self-oscillating mode.

the exhaust duct were acquired. The outlet nozzle diameter used in this case was $7.5 \mathrm{~mm}$, which yields an outlet Mach number of $M \approx 0.47$.

Figure 5 shows the power spectrum of the thermocouple in the combustion chamber at $x=85 \mathrm{~mm}$ and $r=25 \mathrm{~mm}$ with a clear peak at $115 \mathrm{~Hz}$, which is also found in the acoustic signals.

This indicates the convection of so called hot spots with the resonance frequency of the system. To extract the phase relationship the cross-spectral density function is evaluated between the thermocouple and the microphones in the combustion chamber and the exhaust duct, respectively. Figure 6 displays the zoomed cross spectra for the above mentioned self-oscillating resonance case. Considering plane propagation modes the slope in a phasefrequency diagram is inversely proportional to the corresponding propagation speed. The difference between the curves of the two cross spectra in figure 6 is obvious. While the phase between thermocouple and combustion chamber microphone (green) shows a negligible slope according to a high propagation velocity, the phase relation between the thermocouple and the exhaust microphone (black) indicates a much steeper slope related to a distinct lower propagation velocity. Regarding a propagation distance between the thermocouple and the convergent-divergent nozzle of $52.5 \mathrm{~mm}$ the phase relation results in a propagation velocity of approximately $3 \mathrm{~m} / \mathrm{s}$, which matches the mean flow velocity in this region. By comparison the speed of sound in the combustion chamber is as high as $900 \mathrm{~m} / \mathrm{s}$. Thus, the temperature or entropy perturbation is
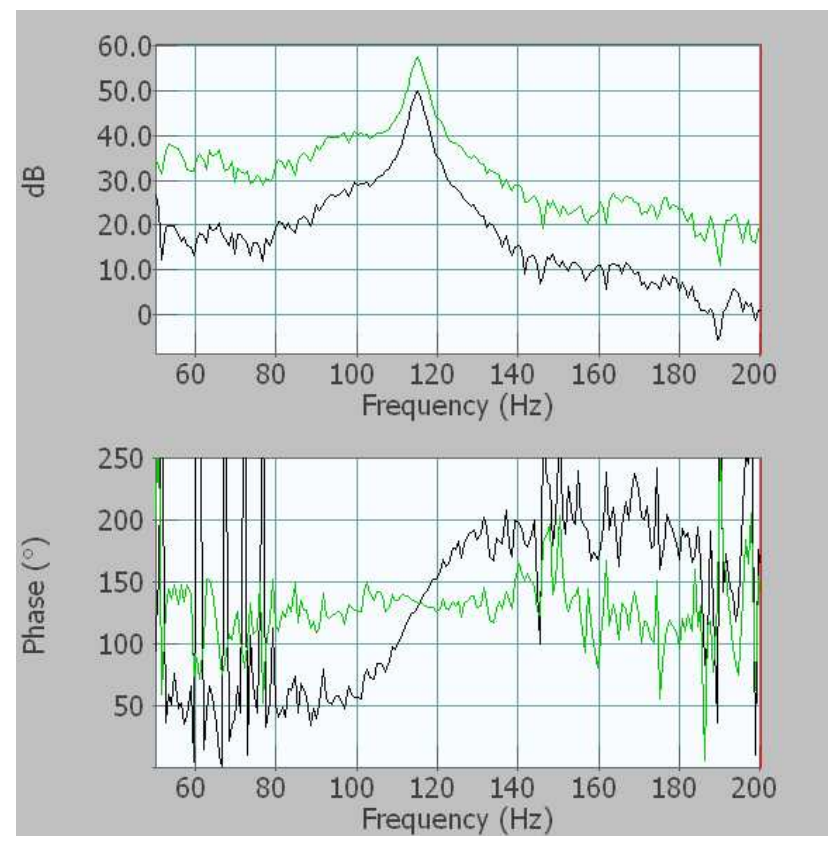

Figure 6. Amplitudes and phases of the cross spectra between thermocouple and combustion chamber microphone (green) and the exhaust duct microphone (black) near to the self-oscillating frequency.

convected with flow velocity from the primary combustion zone passing the thermocouple and accelerated in the outlet nozzle, where the entropy noise generation takes place. The latter is detected by the exhaust duct microphone. In contrast, the phase-frequency relationship between the thermocouple and the combustion 
chamber microphone (green) can be interpreted as the measurement of other combustion noise.

\section{Non-periodical Excitation}

Another method to investigate entropy noise is to operate the combustion rig in a pulse-like excitation mode. The fuel gas supply is modulated using a piezo-electrical mass flux modulator. The pulses are generated by increasing the fuel gas supply for a period of about 17 milliseconds once per second. In the post-processing procedure the microphone and thermocouple signals are phase averaged in reference to the excitation trigger signal. In this setup the thermocouple signal was acquired from the throat position of the convergent-divergent nozzle.
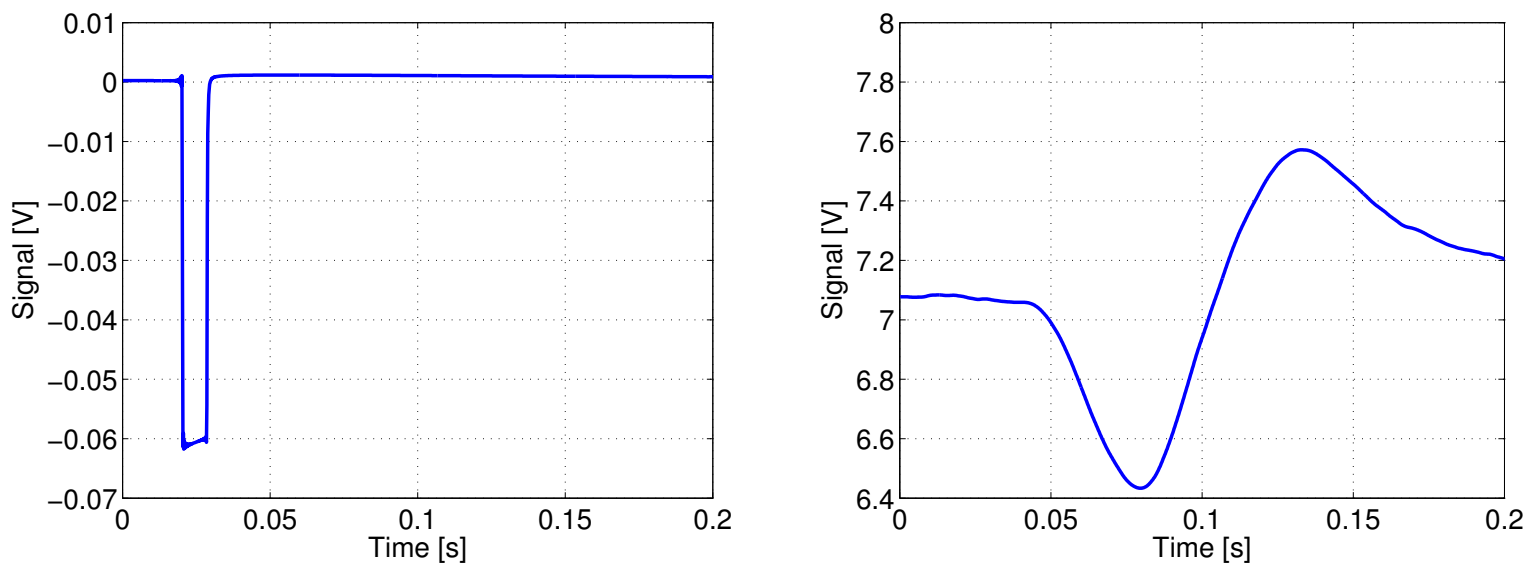

Figure 7. Phase averaged time series of trigger (left) and thermocouple (right) signal in the non-periodical excitation mode.

Figure 7 shows the phase averaged time series of the fuel modulator trigger input on the left and of the thermocouple signal in the outlet nozzle on the right. After a delay period of about 50 milliseconds from the trigger pulse $\left(t_{0}=0.02 \mathrm{~s}\right)$ the thermocouple indicates a distinct temperature modulation in the outlet nozzle with an amplitude of $1.2 \mathrm{~V}$ corresponding to about $100 \mathrm{~K}$. Thus the triggered modulation of the fuel mass flux generates a perturbation of the heat release and with it a convecting entropy wave. The outlet Mach number was $M \approx 0.5$ in this case.
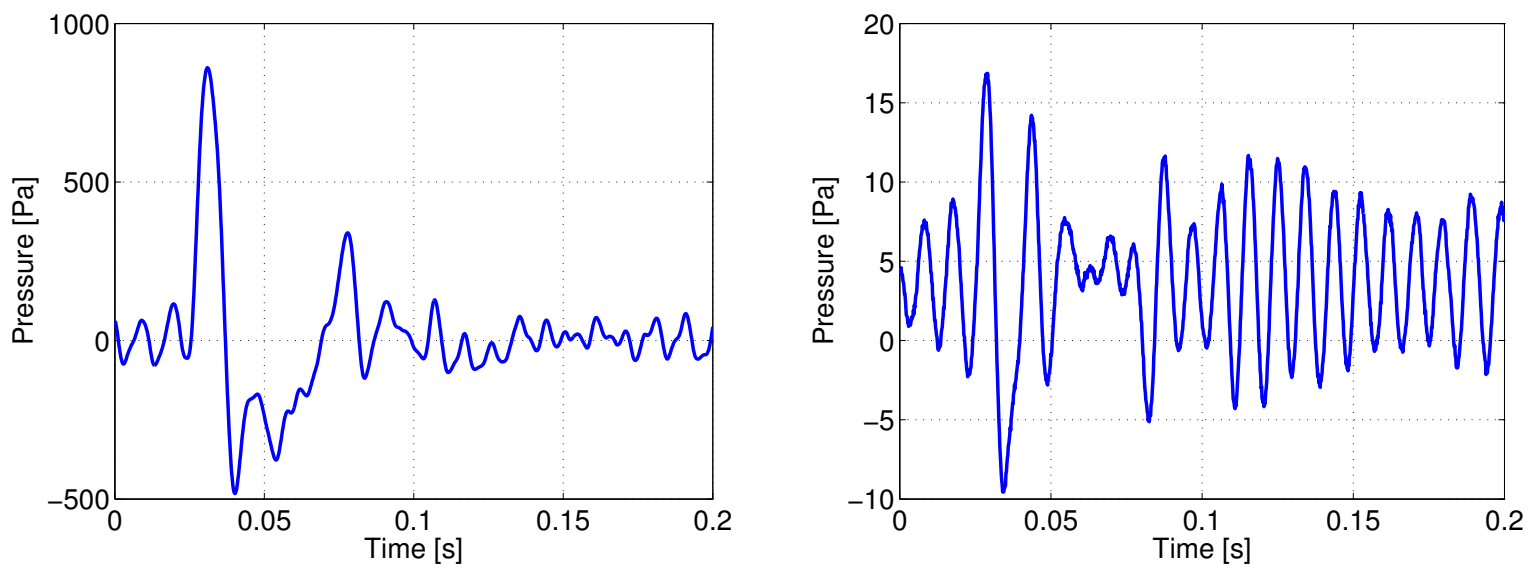

Figure 8. Phase averaged time series of combustion chamber microphone (left) and exhaust duct microphone (right) signal in the non-periodical excitation mode.

The phase averaged microphone signals are displayed in figure 8. Both the combustion chamber (left) and the exhaust duct (right) microphones show an intense pressure pulse shortly after the fuel trigger, which 
corresponds to the direct flame response. The modulation in the fuel mass flux causes a fluctuation in the heat release, which generates the primary propagating sound wave. But the fluctuation in the heat release also produces a downstream convecting entropy wave, which creates a second pressure signature when it passes through the outlet nozzle visible in both microphone signals in figure 8 . The convection time of about $50 \mathrm{~ms}$ fits to the mean flow velocity and the convection path in the combustion chamber. The effect of the two microphones in the combustion chamber and the exhaust duct being out of phase at the second pressure pulse series agrees with the theory. ${ }^{3}$

\section{High-Frequency Entropy Noise}

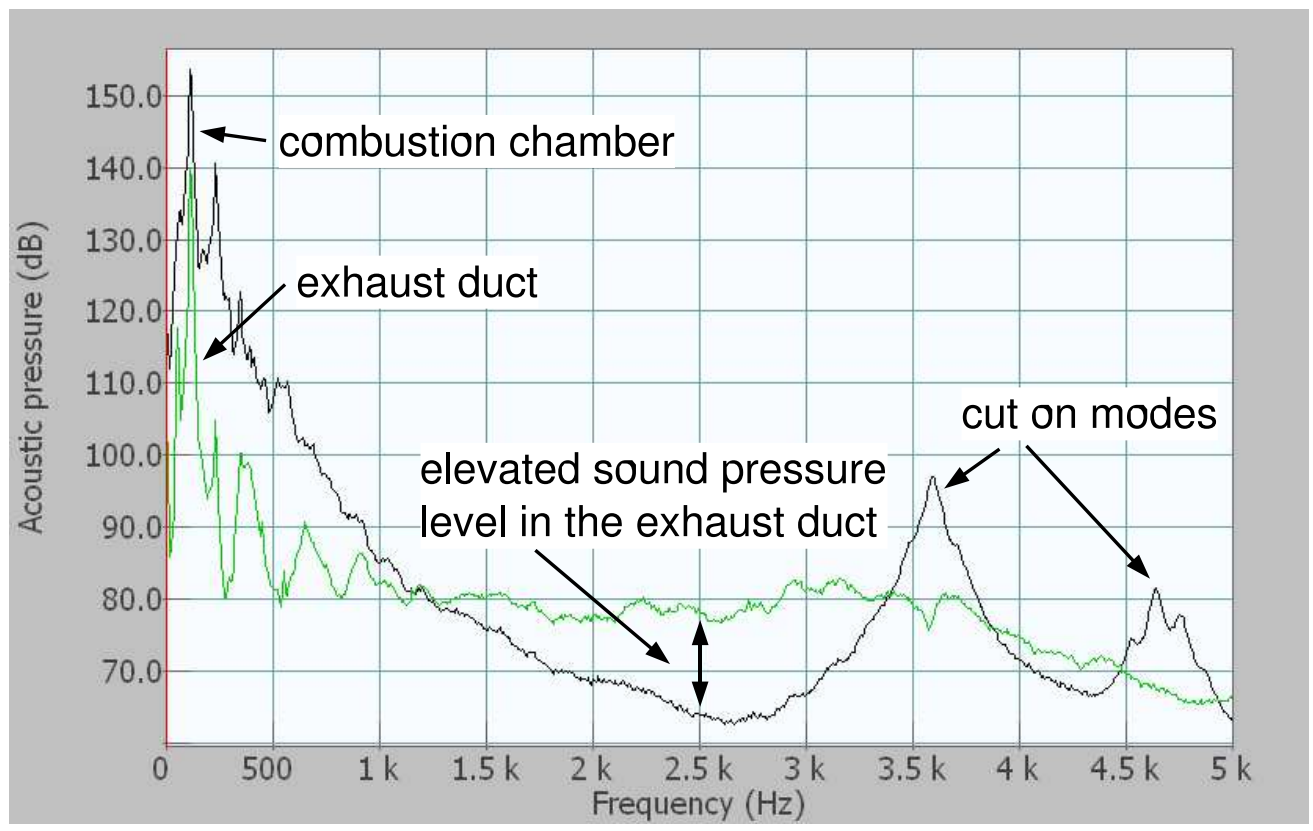

Figure 9. Power spectrum of combustion chamber (black) and exhaust duct (green) microphone up to $5 \mathrm{kHz}$.

A third indication of the existence of entropy noise becomes apparent when the acoustic power spectra of the combustion chamber and the exhaust duct are compared in a broader frequency region up to $5 \mathrm{kHz}$ like in figure 9 . In the frequency band between 1 and $3.5 \mathrm{kHz}$ the sound pressure level of the exhaust duct microphone (green) is clearly elevated above the combustion chamber microphone (black). Interestingly, all exhaust duct microphones at the different axial positions show the same behavior. An estimation of the power of jet noise created by the jet behind the nozzle at the bgeinning of the exhaust duct gives only a minor contribution to the total noise level due to the low mean flow velocity $<2 \mathrm{~m} / \mathrm{s}$. This suggests the assumption that this additional sound pressure level in the exhaust duct is related to small scale entropy fluctuations accelerated in the highly turbulent flow through the combustion chamber outlet nozzle. The spectrum of the microphone in the combustion chamber shows furthermore peaks at the cut-on frequencies of higher order radial modes at 3.6 and $4.7 \mathrm{kHz}$. These modes cannot propagate through the nozzle into the exhaust duct.

\section{Numerical Approach}

A hybrid computation model is used for the numerical simulations. Since it is not reasonable with respect to computation times to resolve both acoustic and convective perturbations in one simulation the flow field including the chemical reaction processes are provided by a URANS-computation. The URANS results then serve as the base flow for the subsequent CAA simulation. In the following the configurations of both methods are outlined first and then the coupling procedures are described. 


\section{A. CFD of Base Flow}

The major task of CFD (Computational Fluid Dynamics) was the numerical simulation of the fluid flow and the related phenomena like turbulence as well as the generation and propagation of coherent structures. In the simulation of reactive flows in a combustion chamber, some additional physical processes, e.g. turbulent mixing of components and chemical reactions have to be considered.

The CAA is looking at the acoustic length and time scales only, in a flow simulated with CFD, disturbances traveling with the speed of sound (acoustic) and those traveling with mean flow velocity (convective) can occur.

However, to resolve both acoustic and convective scales by CFD requires exceptionally large numerical effort and, in general, is not feasible for practical problems. Thus, in the approach adopted here, high Ma-number phenomena are treated in CAA-calculations while the CFD serves for the simulation of the underlying low Ma-number flow field.

Another main issue in simulating fluid flows is, beneath the modeling of reaction in reactive flows, the description of the turbulence. Several approaches exist to calculate turbulent fluctuations of the mean flow quantities, depending on the level modeling.

Using the so called URANS-method (Unsteady Reynolds Averaged Navier Stokes) the turbulent fluctuations will be modeled over the whole energy spectrum of turbulent energy, whereas periodic oscillations are resolved in a time-depend calculation. ${ }^{8}$

In contrast to URANS, LES and DNS methods lack deepness in modeling, and thus they are more universal and may result in more accurate solutions especially when calculating unsteady flows. But because of much higher efficency of URANS methods in contrast to LES or DNS, URANS is used most often in calculating practical problems.

All CFD simulations performed in the present work are based on URANS methods. Most of the time the standard k, $\epsilon-$ Model was used, but also some calculations were made with the RSM-model by Launder et al. ${ }^{9}$ For modeling turbulent combustion a DLR inhouse $\delta$-jpdf-model of $\mathrm{Kunz}^{10}$ and the EDM-model by Magnussen \& Hjertager ${ }^{11}$ was used.

The simulations were performed on an unstructured quadri-

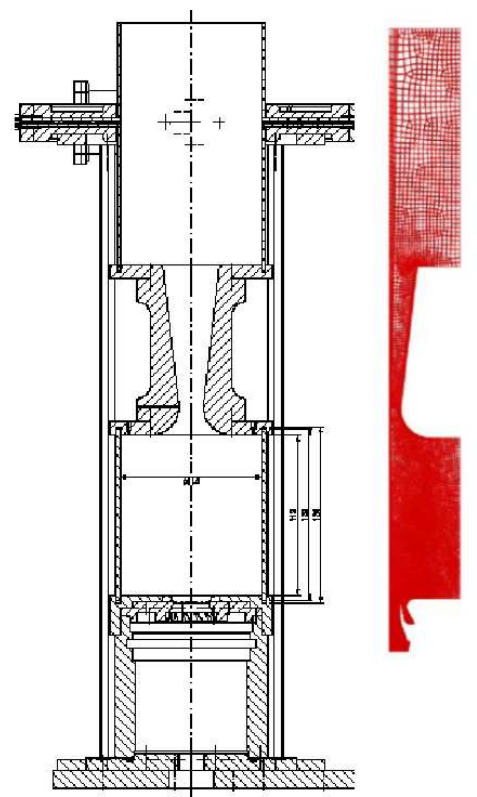

Figure 10. Combustion chamber setup and part of used grid for the URANS calculations.

lateral 2d-grid with about 11000 grid cells. As shown in figure 10, the grid includes the combustion chamber itself as well as the convergent-divergent nozzle structure and a part of the exhaust duct. Because of rotational symmetry a 2-dimensional grid was sufficient.

The air inlets of the computational field were placed immediately behind the radial flow swirlers. The fuel inlet was defined at the fuel injection ports, which were approximated by an annular slot.

The outlet of the computational field was placed in the duct behind the combustion chamber as indicated in figure 10 .

A streamline image and the mean temperature field of the flow field in the combustion chamber is shown in figure 11. The flow field features the typical swirl induced recirculation zone on the center axis of the combustion chamber, which stabilizes the flame in a cone-shaped form. This indicates the maximum region in the temperature distribution.

Various simulation runs were performed in applying different types of boundary conditions at in- and outlets. In the calculations presented here, self-exited combustion oscillations were simulated. For this, total pressure boundary conditions were used at all air inlets.

\section{B. CAA for Acoustic Propagation Modeling}

The CAA-method for the propagation region employs a seven point optimized $(\eta=1.1)$ Dispersion-RelationPreserving (DRP) scheme of fourth order. ${ }^{12}$ The outer three points of the computational mesh are treated by an optimized $(\eta=\pi / 2)$ backward seven point stencil. ${ }^{13}$ The time stepping applies alternating two 

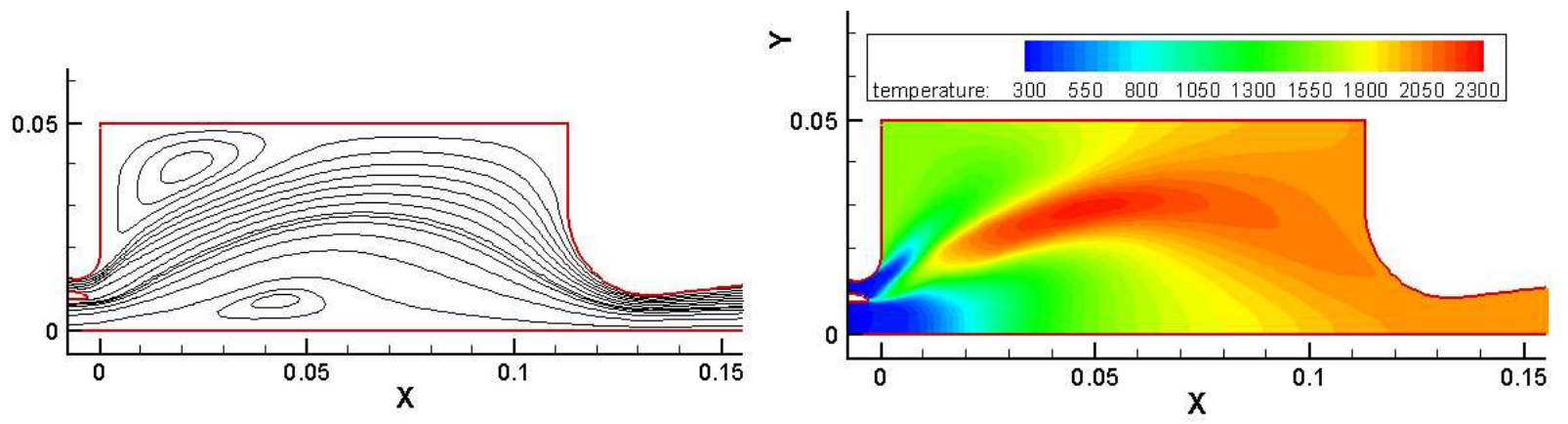

Figure 11. Streamline plot (left) and temperature field (right) in the combustion chamber (CFD results).

fourth order Runge-Kutta schemes with five and six stages. ${ }^{14,15}$ These finite difference methods do not have conservation properties but high order of accuracy and low dissipation and low dispersion.

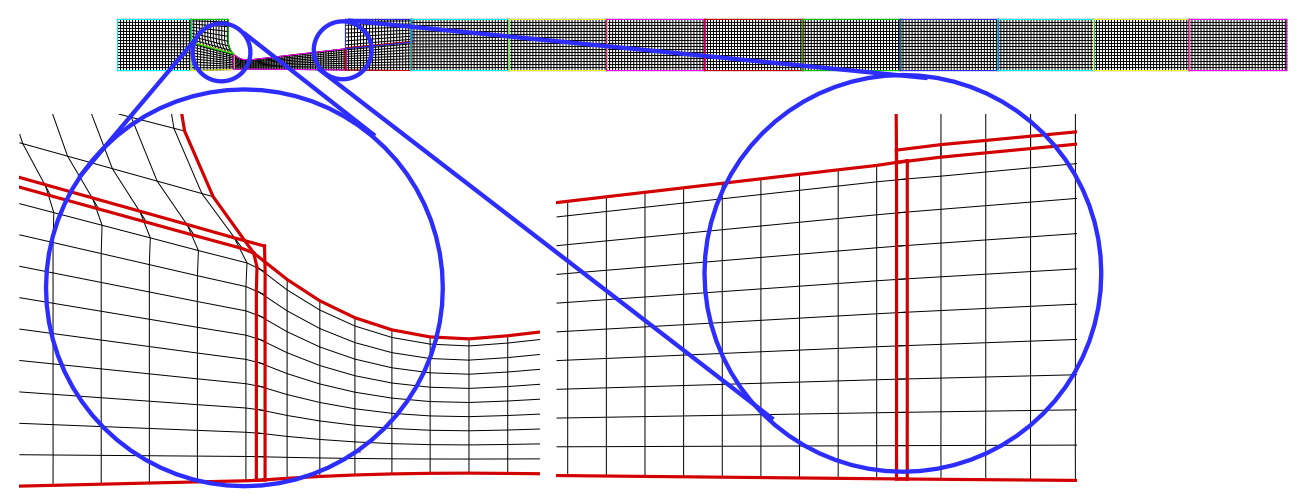

Figure 12. Schematic view of the grid in the CAA domain.

Figure 12 shows a schematic view of the grid used in the CAA simulation with focus points on the inlet and outlet of the convergent-divergent nozzle structure.

\section{Filtering approaches}

Filtering is required to remove the short wavelengths in the unstable range of the discretization schemes, for waves with less then $\approx 5.4$ grid points per wave length (ppw). This band of spatially not sufficiently resolved waves is called short wave component.

In the early years of CAA instead of an explicit filter a selective artificial damping was applied. ${ }^{16}$ This decreases the additional computational time for the filtering to a minimum by combining the time marching and the update of the filtered field. The short wave component of one time step, is filtered out after the calculation of the spatial derivatives. For this reason the pollution by short wave components increases with the steady entry of energy to this band by the non-linear Euler-equations. Therefore, this method is unstable especially for long computations.

The idea is now to use a filter placed outside the inner time stepping loop. Then the field used to calculate the derivatives is free of short waves. The filter is chosen to be explicit, as the exact implicit solution requires more computational time for large systems.

Different filter algorithms are applied ${ }^{17,18}$ and their characteristics are investigated. A small additional dissipation is applied by the filtering schemes of seven points wide in the stable range of the DRP scheme, which is somewhat reduced by the optimization. For higher point numbers the filter characteristics show a more steep growth from pass-band to the blocked wavelength and the pass band is extended to the required 
5.4 points per wavelength. The filter should not be optimized for long time calculations for the reason that the extended pass-band range shows a declined dissipation.

In a numerical experiment each of the filters were applied with the same filtering amplitude to different harmonic plain waves. The results show strong dissipation of the seven point filters as expected. The eleven point filters reduces the dissipation. The optimized eleven point filter ${ }^{18}$ reorders the curves in the way, that the 11 ppw curve shows less dissipation than the 16 and 32 ppw ones. The usable stable range of the overall scheme is extended down to $5 \mathrm{ppw}$ by this filter at the expense of little higher losses over the whole range than the standard filter shows. The most satisfactory solution is obtained using an eleven point standard filter of Bogey. ${ }^{18}$ The boundary regions are treated by smaller symmetric filter stencils.

\section{Wall boundary condition}

The wall boundary condition is implemented according to Tam. ${ }^{13}$ The change of the normal velocity from one time-step to the next time-step with a vanishing ghost pressure is used as condition to calculate the pressure opposed by the wall in the virtual ghost point. The ghost point does not need to be stored. As the full or even the modified governing equations in the sponge and PML layers are used, the ghost point implementation is independent of the used model (LEE or PENNE) and compatible to any kind of boundary condition.

\section{Inlet and outlet conditions}

A modified PML boundary condition is applied as outlet boundary condition. It is derived based on Hu's ${ }^{19}$ PML using a cylindrical coordinate system. The PML is formulated in un-split variables in order to reduce the overhead of variables in the inner computational domain. The parts of the modified Euler-equations in the PML can be identified as the original Euler-equations, a sponge term, a correction for waves passing normally to the sponge layer and a correction for the convective terms on these waves. The PML is applied in the outlet duct of the combustion chamber. The inlet is reproduced by a simple sponge layer of dissipative medium without perfectly matched characteristics. This technique is used to couple the sound source introduced by the flame elastically to the CAA-domain. 


\section{Coupling Strategies}

The coupling strategies between CAA and CFD can be divided into a three step concept:

- First, a steady RANS output serves as an input for the CAA simulation where it is superposed with an artificial entropy perturbation located in the primary combustion zone. The propagation and sound generation of this convected entropy wave is then calculated in the CAA code.

- In the second approach, an unsteady RANS solution forced by periodic modulation of the fuel gas supply or by a modulated pressure fluctuation in the outlet plane is evaluated in the CAA simulation.

- Finally, a self-excited URANS calculation is processed using the CAA method.

\section{Results}

The first coupling scheme is particularly suited to demonstrate the effect of sound generating entropy fluctuations in a accelerated mean flow. Figure 13 shows color plots of three time steps of such a convecting entropy wave (upper half) through the outlet nozzle and the evolving sound pressure. In the first two time steps, before the entropy waves reach the outlet nozzle, no sound pressure is generated (lower half of each time step plot). But when the entropy wave passes the outlet nozzle (third time step) an enormous sound pressure amplitude is released.

A comparison between experimental data and CAA results of the URANS solutions is presented in figure 14. The power spectra of the sound pressure levels at the first axial position in the exhaust duct $(x=338 \mathrm{~mm})$ are displayed for the experiment, the CAA based on a forced URANS and the CAA based on a self-excited URANS. In the forced URANS case the pressure in the outlet conditions has been modulated by the experimentally acquired fundamental oscillating frequency of about $90 \mathrm{~Hz}$, which explains the similarity at this frequency. However, the tendency of the sound pressure level to decline in the frequency range between 100 and $2000 \mathrm{~Hz}$ is captured quiet well by the CAA simulation of the forced URANS case.

The fundamental oscillating frequencies of the combustion system with $90 \mathrm{~Hz}$ in the experiment and $383 \mathrm{~Hz}$ in the numerical computation of the URANS results do not match well. This is based on the fact that the URANS simulation does not cover the entire exhaust duct and the plenum system of the combustion chamber (see figure 10).

\section{Conclusion}

The generation of entropy or indirect noise in a model combustor test rig is experimentally proven. The presented work is a good example of cross-linking experimental studies and numerical simulations. The numerical URANS computation shows the significant development of convecting entropy perturbations in a combustor flow. Through successful coupling of the URANS and CAA methods the entropy induced sound generation is simulated by the CAA code. Two different kinds of URANS simulations have been applied, a self-excited and forced case. Since the self-excited case did not match the fundamental oscillating frequency of the experimental data, the forced URANS was driven by an outlet pressure fluctuation of the experimentally acquired dominating frequency. In result the CAA simulation using the forced URANS solution shows a good agreement to the experimental data. However, with regard to further investigations one 


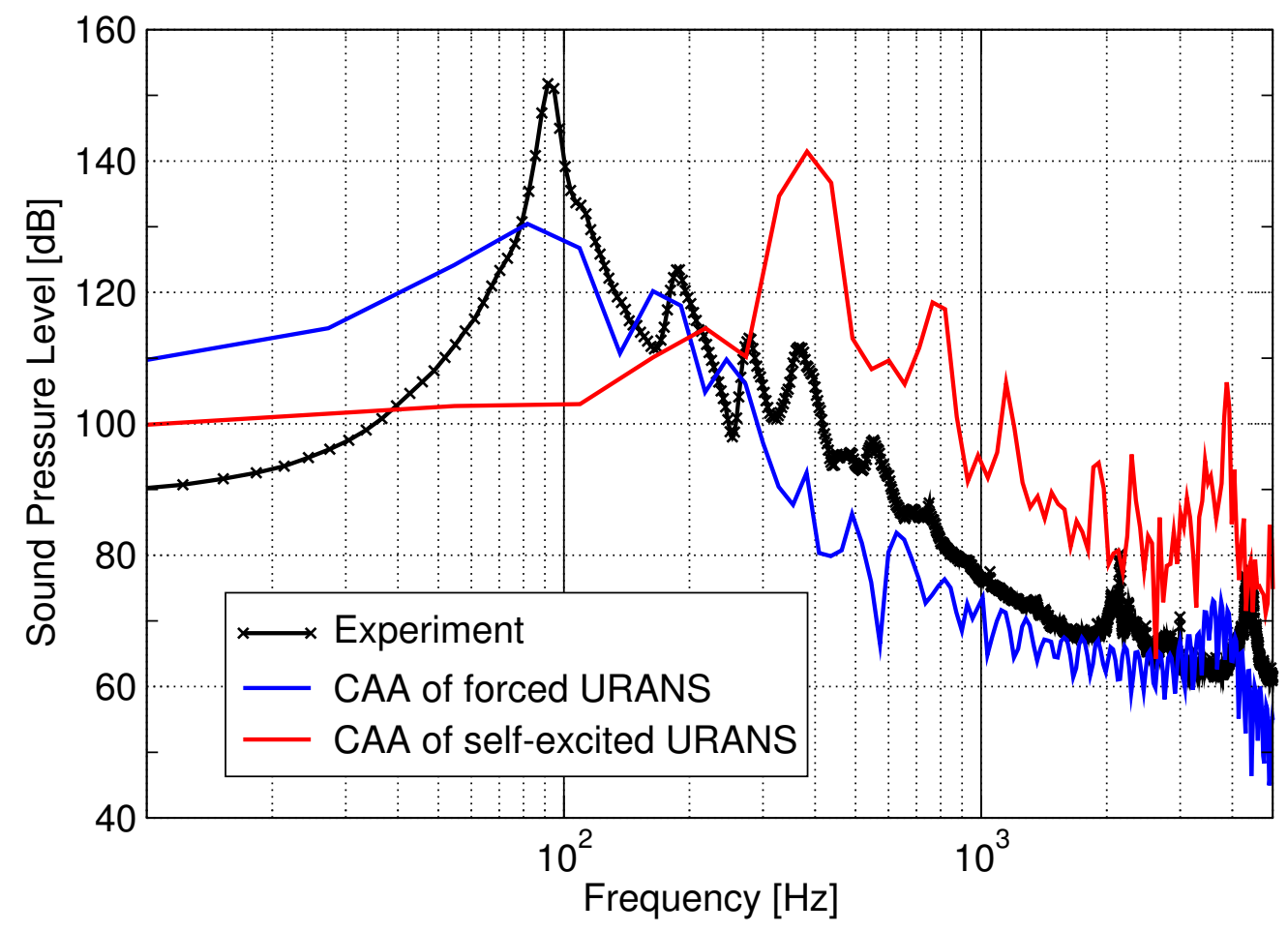

Figure 14. Comparison of numerical data (blue and red) and experimental results (green). Power spectrum of the sound pressure level at the first axial microphone position in the exhaust duct.

goal is a better compliance of the self-excited URANS by improving the boundary conditions. Furthermore, after defining and improving the interfaces between the two numerical methods and in addition to the experimental approach, a profound quantitative analysis of the entropy wave amplitudes and scales with respect to the noise generation at different operating points needs to be accomplished.

One especially interesting experimental result is the generation of indirect combustion noise in the outlet section of the combuster at high frequencies, which are generally not considered to be combustion related. This finding could indicate the possibility of a substantial noise generation at high frequencies in the turbine stages due to entropy fluctuations within the fluid flow. This could mean that a substantial part of broadband core noise of aero-engines at high frequencies is related to the combustor and not to the turbine. This noise generation mechanism would apply in each blade row of a turbine as already suggested by Cumpsty \& Marble. ${ }^{20}$ The conclusion is that future combustors have to provide a uniform entropy distribution at the outlet of the combustion chamber. Premixed combustors may have an advantage in this respect, although they are known to be prone to low-frequency combustion instability.

\section{Acknowledgment}

The authors gratefully acknowledge the financial support by the German Research Foundation (DFG) through the Research Unit FOR 486 "Combustion Noise".

\section{References}

${ }^{1}$ A.P. Dowling, A. P., "Acoustics of unstable flows," Theoretical and Applied Mechanics, edited by T. Tatsumi, E. Watanabe, and T. Kambe, Elsevier, Amsterdam, 1996, pp. 171-186.

${ }^{2}$ Truffaut, J.-M., Searby, G., and Boyer, L., "Sound emission by non-isomolar combustion at low Mach numbers," Combust. 
Theory Modelling, Vol. 2, 1998, pp. 423-428.

${ }^{3}$ Marble, F. E. and Candel, S. M., "Acoustic disturbances from gas non-uniformities convected through a nozzle," J. Sound Vibration, Vol. 55, No. 2, 1977, pp. 225-243.

${ }^{4}$ Muthukrishnan, M., Strahle, W. C., and Neale, D. H., "Separation of Hydrodynamic, Entropy, and Combustion Noise in a Gas Turbine Combustor," AIAA Journal, Vol. 16, No. 4, 1978, pp. 320-327.

${ }^{5}$ Shenoda, F. B., Reflexionsarme Abschlüsse für durchströmte Kanäle, Dissertation, Technische Universität Berlin, 1973.

${ }^{6}$ Enghardt, L., Zhang, Y., and Neise, W., "Experimental verification of a radial mode analysis technique using wall-flush mounted sensors," 137th Meeting of the Acoustical Society of America, Berlin, October 1999, pp. 15-19.

${ }^{7}$ Maier, R., Zillmann, J., Roure, A., Winninger, M., Enghardt, L., Tapken, U., Neise, W., Antoine, H., and Bouty, E., "Active Control of Fan Tone Noise from Aircraft Engines," 7th AIAA/CEAS Aeroacoustics Conference, No. 2001-2220, Maastricht, 2001.

${ }^{8}$ Noll, B., Schütz, H., and Aigner, M., "Numerical simulation of high-frequency flow instabilities near an airblast atomizer," ASME Turbo Expo 2001, No. GT2001-0041, ASME, 2001.

${ }^{9}$ Launder, B., Reece, G., and Rodi, W., "Progress in the development of a Reynolds-stress turbulence closure," J. Fluid Mech., Vol. 68, 1975, pp. 537-566, Part 3.

${ }^{10}$ Kunz, O., PDF-Simulation von Verbrennungsvorgängen in praxisnahen Brennkammern, Dissertation, Fakultät Luftund Raumfahrttechnik der Universität Stuttgart, 2002

${ }^{11}$ Magnussen, B. and Hjertager, B., "On mathematical modelling of turbulent combustion with special emphasis on soot formation and combustion," 16th Intern. Symposium on Combustion, 1976.

${ }^{12}$ Tam, C. K. W. and Webb, C., "Dispersion-Relation-Preserving Finite Difference Schemes for Computational Aeroacoustics," J. Comp Phys., Vol. 107, No. 2, August 1993, pp. 262-281.

${ }^{13}$ Tam, C. and Dong, Z., "Wall Boundary Conditions for High-Order Finite Difference Schemes in Computional Aeroacoustics," Theoret. and Comput. Fluid Dynamics, Vol. 6, No. 6, 1994, pp. 303-322.

${ }^{14} \mathrm{Hu}, \mathrm{F}$., "On Absorbing Boundary Conditions for Linearized Euler Equations by a Perfectly Matched Layer," J. Comput. Phys., Vol. 129, 1996, pp. 201-219.

${ }^{15}$ Stanescu, D. and Habashi, W., "2N-storage Low-dissipation and Low-dispersion Runge-Kutta Schemes for Computational Aeroacoustics," J. Comput. Phys., Vol. 143, No. 2, 1998, pp. 674-681.

${ }^{16}$ Tam, C. K. W., Webb, C., and Dong, T. Z., "A Study of Short Wave Components in Computational Aeroacoustics," Journal of Computational Acoustics, Vol. 1, March 1993, pp. 1-30.

${ }^{17}$ Vasilyev, O., Lund, T., and Moin, P., "A General Class of Commutative Filters for LES in Complex Geometries," J. Comput. Physics, Vol. 146, 1998, pp. 82-104.

${ }^{18}$ Bogey, C. and Bailly, C., "A family of low dispersive and low dissipative explicit schemes for flow and noise computations," J. Comput. Physics, Vol. 194, 2004, pp. 194-214.

${ }^{19} \mathrm{Hu}, \mathrm{F} .$, "A Stable Perfectly Matched Layer For Linearized Euler Equations In Unsplit Physical Variables," J. Comput. Phys., Vol. 173, 2001, pp. 455-480.

${ }^{20}$ Cumpsty, E. and Marble, F., "The interaction of entropy fluctuations with turbine blade rows; a mechanism of turbojet engine noise," Proc. Roy. Soc. London A, Vol. 357, 1977, pp. 323-344. 